\title{
CASE STUDY OF ENGINEERING RISK IN AUTOMOTIVE INDUSTRY
}

\author{
Dan Mihai POPA \\ Politechnica University, Timisoara
}

\begin{abstract}
:
The primary objective of this paper is to show where the engineering of risk management is placed and how its implementation has been tried in multinational companies in automotive industry from Romania. A large number of companies don't use a strategy to avoid the engineering risk in their design products. The main reason is not because these companies haven't heard about standards for risk management such as ISO 31000; the problem is that the business units which were summed up, have just set up a risk list at the beginning of the project, without any follow up. The purpose of this article is to create an implementation risk tracking in automotive industry companies in Romania, due to a change request from customers according to supply companies within the quality process, in the research and development phase.
\end{abstract}

Key words: engineering of risk management, engineering risk, risk management, risk assessment

\section{INTRODUCTION}

Modern companies have to thrive in a very changeable and rapidly developing market where competition is one of the constant elements of the market. In such an environment, enterprises should continuously adjust their strategy to suit the current situation in the market and forthcoming threats and opportunities [1]. In this case study we are going to talk about radar products, these products are used more and more in the automotive industry, at the same time being requested by the EuroNCAP. A project is based on safety approval standards for both drivers and passengers. The 5-star safety rating criteria are granted and based on the endowment with sensors produced in the ADAS (Advance Driving Assistance Systems) departments of the companies operating such solutions.

In a brief introduction of this short chapter, we are considering the EuroNCAP rules for increasing passenger protection. We propose to follow the statistics that underlay this brief case study. The information in this case studio was taken from the statistical website: Statista [2]. Several statistics are gathered as follows: the main sensory companies in Germany based on revenue (expressed in millions of euro) refer to these companies because they have locations in Romania, both for research and production development, as illustrated in Figure 1.

As we mentioned before, as it can be seen on the statistics from Figure 1, these leading German companies are also based in Romania.

The industrial development in Romania includes ADAS sector, a sector which grows up in every year. The main projects are focused on the bracket projects in sensors. In this case study we are talking about one project for bracket radar sensor, the type of radar sensor that is an SRR (Shor Range Radar) sensor.

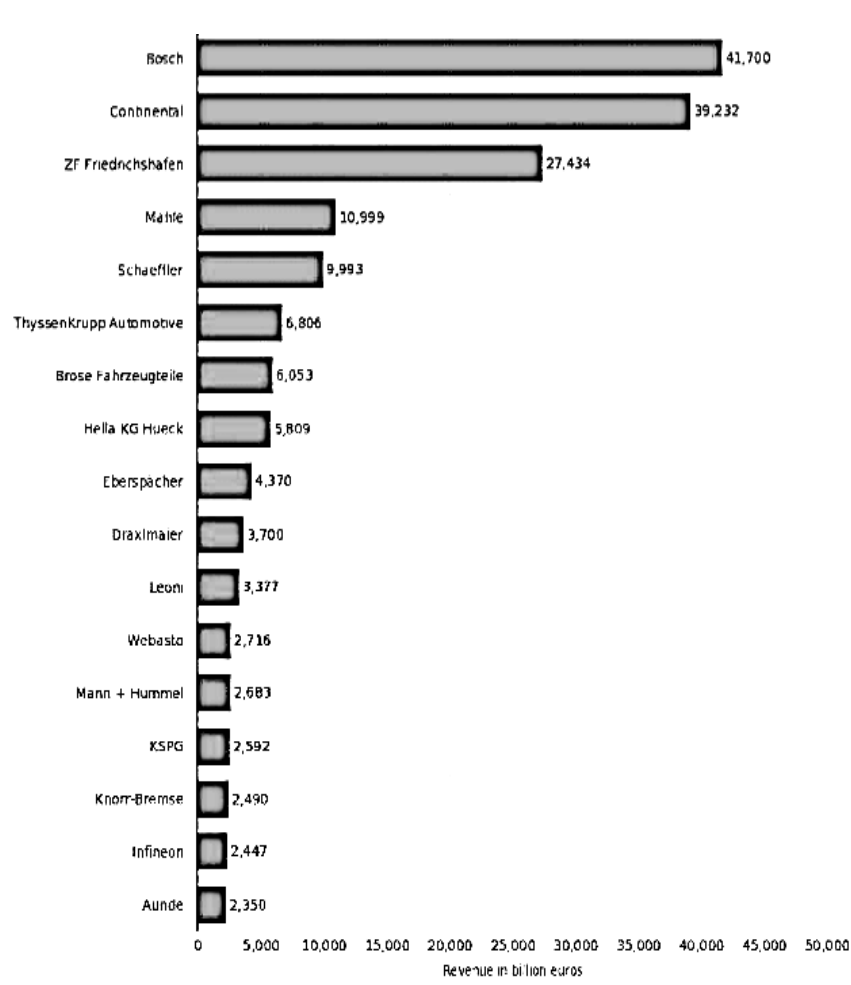

Fig. 1 Leading German automotive suppliers in 2015, based on revenue (in million euro)

Source: [2].

\section{METHODOLOGY OF RESEARCH}

In case study the engineering risk management is directly related to ISO 31000 standard for identifed risk and monitorized risk. The scheme for this process as ilustrated in Figure 2. 


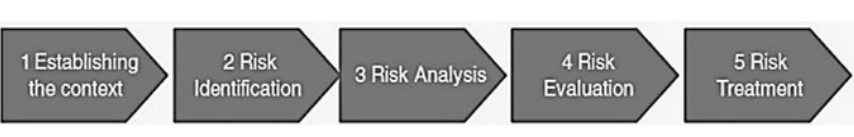

Fig. 2 Risk management process

Source: [3].

We have mentioned the correlation of the process between the customer and the supplier in each stage of the process in a sensor support project, after all steps, even a test of the product quality in the laboratory. After we signed the final quality report with customer, they wanted to come up with a change request. What's happened? On the quotation stage, this risk was not considered because a risk management plan was not taken in consideration, people are aware of it, but there is no management of it. There is only a list of risk actions that are not treated or drawn completely and which are kept in the system.

The technical point of view for this action has been to have daily meetings to keep track of this risk. The change request came from customer because they wanted to remove one fixation point from bracket which has been designed for an SRR sensor; this bracket was designed and was meant for 3 fixation points not for 2 fixation points. The customer was informed about implication on this change and they requested quotation for another product validation report, another product validation report means generating another final quality report and additional costs, test laboratory was booked; after this, according to the final quality report the actions for D-FMEA (Design - Failure Mode and Effect Analysis) was closed and reported to the customer.

Methodology and instruments for this blocking point was initiated with an FEA (Finit Element Analysis) simulation.

\section{MOUNTING AND GUIDELINE FOR SRR}

\section{SRR Overview}

The SRR sensor is a $24 \mathrm{GHz}$ radar sensor for short range applications. This overview is meant to provide a general guideline for sensor mounting and integration into the subject vehicle, but makes no claims of being complete.

The supplier of this product is Continental and supplier must confirm suitable radar performance of the overall system (Radar integrated in the target platform) and thus confirm final mounting position [4].

\section{SRR Mounting Requirements}

The sensor can be mounted in normal or flipped orientation. I. e. each sensor can be mounted on the left or right hand side of the vehicle, connector facing downwards or upwards direction (Defined in Vehicle specification) but in same direction on both sides of the target vehicle platform. The sensor must be protected from direct impact of water/ mud/snow.

The mounting window depends on the function which is realized with the SRR320 sensor. These requirements are given below: $\alpha$ (alpha), $\beta$ (beta) and $\gamma$ (gamma) angles are defined in Figure 3: Definition of angles. Sense of rotation of angles (sign) is defined according to the right hand rule [4].

Remarks:

1. For LCA (Lane Change Assist) function, a mounting alpha angle of $50^{\circ}$ is needed.

2. The mounting position values and tolerances are specified with the experience of SRR220 project developments and SRR320 predevelopment studies. The values are nevertheless not completely validated with vehicle tests and are subjects to final adjustments within final sensor validation.

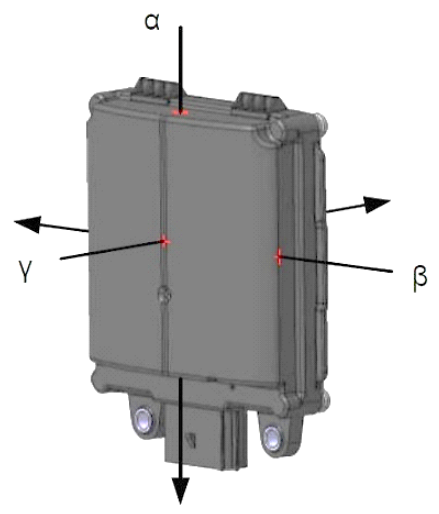

Fig. 3 Definition of angles for SRR 320 sensor Source: [4].

\section{Mounting Tolerances}

The azimuth mounting tolerance $\Delta \alpha$ must not exceed the value given in Table 1 [4]. In this overall tolerance, the following contributions are included:

1. Drive axis-vehicle body,

2. Vehicle Body-Bracket,

3. Bracket-Sensor.

The mechanical mounting tolerance in elevation (roll angle) $\Delta \beta$ must not exceed the value given in Table 1 [4]. In this overall tolerance, the following contributions are included:

1. Vehicle Body-Bracket.

2. Bracket-Sensor.

The mechanical mounting tolerance for the pitch angle $\Delta \psi$ shall not exceed the value given in Table 1 [4].

Table 1

SRR mounting orientation requirements

\begin{tabular}{cccc}
\hline Points & Parameter & Adjustment & Tolerances \\
1 & $\Delta x(x-$ coordinate $)$ & $300 \mathrm{~mm}$ & $+/-200 \mathrm{~mm}$ \\
2 & $\Delta y(y$ - coordinate $)$ & $100 \mathrm{~mm}$ & $+/-50 \mathrm{~mm}$ \\
3 & $H \_z$ - coordinate $)$ & $600 \mathrm{~mm}$ & $+250 /-100 \mathrm{~mm}$ \\
4 & $\alpha$ & $25,27,30,37,39,40^{\circ}$ & $+/-3^{\circ}$ \\
5 & $\beta$ & $0^{\circ}$ & $+/-3^{\circ}$ \\
6 & $\gamma$ & $0^{\circ}$ & $+/-3^{\circ}$ \\
\hline
\end{tabular}


In this overall tolerance, the following contributions are included:

1. Vehicle Body-Bracket.

2. Bracket-Sensor.

Attention must be paid to the facts that:

If not otherwise agreed in contract specification, the given mounting tolerances do not take into consideration additional misalignments imposed by load changes of the subject vehicle. I.e. Additional tolerances imposed by load of passengers and/or luggage etc.

Additional Misalignment, especially in elevation and pitch (by load changes) cannot be compensated by any sensor means [4].

The mounting window and mounting tolerances given in this document have been determined theoretically and should be verified by tests.

\section{Situation of installation}

In general terms, for a proper sensor performance the area in front of the sensor field of view (FOV - Field of view) needs to be kept free of any materials or objects that may disturb the millimeter wave emission characteristics.

As this cannot be achieved under all circumstances, below sections provide a guidance to minimize impact of vehicle parts in the FOV of the sensor.

\section{Test coverage}

To limit the impact of the particular situation of installation on the system performance, verification tests must be conducted as early as possible. Sensor supplier can offer the testing support, but testing effort needs to be planned and scheduled thoroughly as resources are limited.

Test planning and scheduling shall be agreed with sensor supplier at the beginning of a project (car platform development). To keep test effort at a reasonable level, tests shall be planned and conducted only when test results can be applied to the final platform. Appropriate tests are defined within most of the following sections.

\section{Distance and Angle}

According to the mounting position of the sensor for a specific platform, the distance between the sensor front cover and the bumper (secondary surface) shall be designed large enough to avoid mechanical interferences, caused by thermal geometry changes or mechanical stress (vibrations).

Bumper and sensor shall not be in contact. There must be at a minimum distance that ensures the free space between sensor and bumper is not blocked by dirt.

An exact parallel orientation of the bumper (secondary surface) with respect to the sensors front surface which would cause a direct back reflection of the radar radiation into the sensors antenna shall be avoided.

\section{RESULTS OF RESEARCH}

We talked about mounting and guidelines for sensor needing an overview about design bracket and to understand the requirements for FEA simulation. On the Figure 4 the situation of fixed points for bracket and sensor is illustrated, as in this situation was used Ansys simulation software under licensed powered by Continental Automotive.

There was a request to give up the clip-up on the third point of attachment as it is a special bolt that is screwed into the production line that is automatic and the production line usually assembles even four different platforms function of production requirements. The screw of the third fixed point was different from the other customer platforms. To avoid another change of bit screw and gain time on the assembly line, it is desirable to drop this screw. The customer requests a new quotation for laboratory test analysis for this situation to simulate new situation on the machine only with two fixed points.

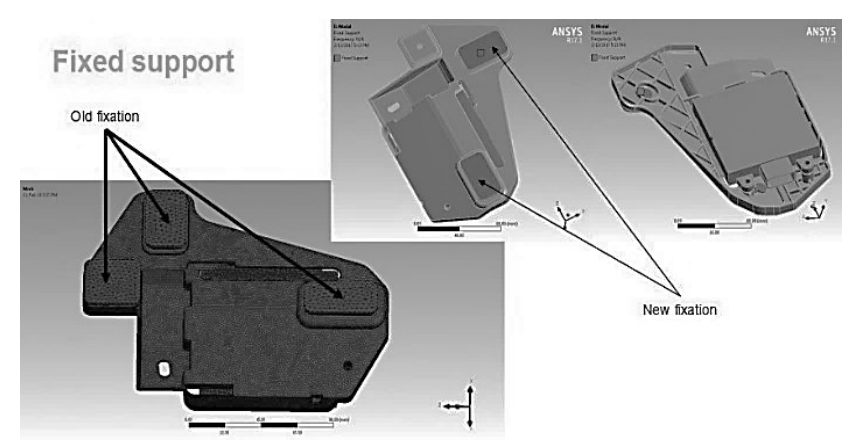

Fig. 4 Comparison of fixed support with 2 vs. 3 points

Simulations required from this FEA analysis was provided from customer to performed harmonic and shock vibration with inputs from customer according with their owned specification. Results as illustrated on the Figure 5.

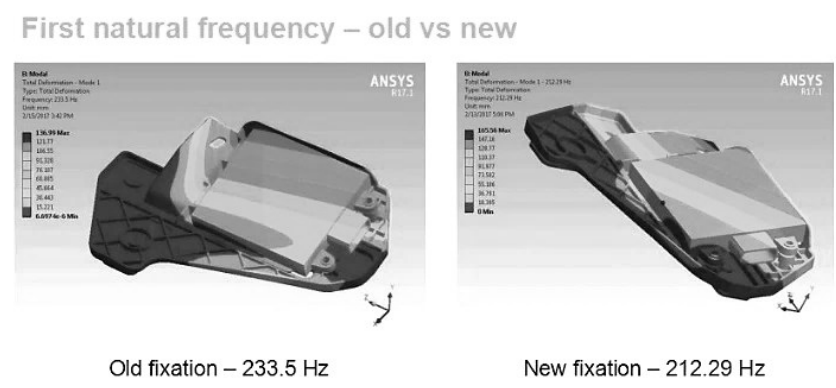

Fig. 5 First natural frequency after FEA simulation

In order to avoid the problems in quote phases we needed to try an implementation as engineering of risk management in Supply Chains has gained increasing attention over the last years.

At the same time, the pressure to consider social issues in global and interconnected supply chains has risen as well. These issues pose risk to supply chains since their occurrence may lead to a bad reputation that would, in its turn, cause adverse stakeholder reactions [5].

\section{CONCLUSIONS}

Based on results and experience design department and system architect from ADAS department we concluded, after a good evaluation report of FEA, that we needed to take in consideration every scenario. Our team concluded:

1. The first natural frequencies for both fixation types are above on interval $5-200 \mathrm{~Hz}$ sinusoidal vibration domain and stress values from harmonic vibrations are low.

2. The rest of the stress values for shock are below the yield limit of the material and occur in the same location, the limit was okay.

3. The gap dimension in between the bracket and sheet metal will be variable and more likely produced an unexpected vibration (e.g. rattle noise) which is not okay.

4. Additional testing with the whole assembly (bracket and sensor and real geometry of sheet metal) should be 
performed to validate the feasibility of the new proposed fixation of the bracket.

5. Radar performance can be affected by these additional vibrations; we take into consideration a system level detection test, more exactly, a functional test for the radar while running the random vibration profile which was defined which can affect the radar performance to required object detection in the new fixation and with the additional unpredictable vibrations taken into consideration.

\section{ACKNOWLEDGEMENT}

This article is based on results of the project bracket SRR320FD17_U55X financed from Approval Budget of Project brackets by Continental Automotive Romania SRL

\section{REFERENCES}

[1] J. Jurczak, "Intellectual Capital Risk Management for Knowledge-Based Organizations", in Risk Management in Public Administration, K. Raczkowski, Ed. Cham: Springer International Publishing AG, 2017, pp. 257-282.

\section{Dipl. Eng. Dan Mihai Popa}

Politehnica University of Timisoara

Bd. Mihai Viteazu no. 1, 300222, Timisoara, ROMANIA

tel.: +40 256403591

e-mail: danmihai.popa@student.upt.ro
[2] Berylls Strategy Advisors, "Leading German automotive suppliers in 2015, based on revenue (in million euros)", Berylls Strategy Advisors GmbH, Munich, Germany, 2015.

[3] Y. Wijnia, "Asset Risk Management: Issues in the Design and Use of the Risk Matrix", in Engineering Asset Management and Infrastructure Sustainability, J. Mathew, L. Ma, A. Tan, M. Weijnen and J. Lee, Eds. London: Springer-Verlag, 2011, pp. 1043-1059.

[4] ADC Continental Automotive Systems GmbH, SRR 320 Mounting and Guidelines, ADC Continental Automotive Systems GmbH, Lindau, Germany, 2014, pp. 4-16.

[5] K. Zimmer, M. Fröhling, P. Breun and F. Schultmann, "Assessing social risks of global supply chains: a quantitative analytical approach and its application to supplier selection in the German automotive industry", Journal of Cleaner Production, vol. 149, pp. 96-109, 2017. 